Full Length Article

\title{
Correlation between vertebral bone microstructure and estimated strength in elderly women: An ex-vivo HR-pQCT study of cadaveric spine
}

\author{
Shuta Yamada ${ }^{a}$, Ko Chiba ${ }^{\mathrm{a}, *}$, Narihiro Okazaki ${ }^{\mathrm{a}}$, Makoto Era ${ }^{\mathrm{a}}$, Yuichiro Nishino ${ }^{\mathrm{a}}$, \\ Kazuaki Yokota ${ }^{\mathrm{a}}$, Akihiko Yonekura ${ }^{\mathrm{a}}$, Masato Tomita ${ }^{\mathrm{a}}$, Toshiyuki Tsurumoto ${ }^{\mathrm{b}}$, Makoto Osaki ${ }^{\mathrm{a}}$ \\ ${ }^{a}$ Department of Orthopedic Surgery, Nagasaki University Graduate School of Biomedical Sciences, Japan \\ ${ }^{\mathrm{b}}$ Department of Macroscopic Anatomy, Nagasaki University Graduate School of Biomedical Sciences, Japan
}

\section{A R T I C L E I N F O}

\section{Keywords:}

High-resolution peripheral quantitative computed tomography (HR-pQCT)

Bone microstructure

Estimated bone strength

Vertebral body

\begin{abstract}
A B S T R A C T
Purpose: A vertebral fracture is the most common complication of osteoporosis, and various factors are involved in its occurrence. The purpose of this study was to investigate the role of trabecular and cortical bone microstructure on vertebral strength using high-resolution peripheral quantitative computed tomography (HR-pQCT). Methods: Three female cadaveric spines were investigated (average age: 80.3 years). The whole spine (T1-L4) was scanned by second-generation HR-pQCT at a voxel size of $60.7 \mu \mathrm{m}$. Bone microstructure analysis and micro finite element analysis were performed after excluding the upper and lower endplates and posterior elements of a total of 48 vertebrae. Correlations between trabecular and cortical bone microstructure parameters and estimated vertebral strength were analyzed by univariate and multivariate regression models.

Results: Cortical thickness (Ct.Th) and trabecular thickness (Tb.Th) were strongly correlated with estimated failure load on univariate analysis ( $r=0.89,0.82)$. Trabecular volumetric bone mineral density (Tb.vBMD), bone volume fraction (BV/TV), trabecular number (Tb.N), and Ct.Th were correlated with estimated failure load on multivariate regression analysis.

Conclusions: It was suggested that, in addition to trabecular bone (Tb.vBMD, BV/TV, Tb.N), cortical bone (Ct.Th) contributed significantly to vertebral strength in elderly women.
\end{abstract}

\section{Introduction}

Osteoporotic vertebral fractures decrease daily activity levels and quality of life for elderly people and increase their mortality rate. It is reported that the incidence of vertebral fractures is five times higher in women than in men [1-3]. Additionally, the incidence of vertebral fractures in women in their $80 \mathrm{~s}$ is four times that of women in their $60 \mathrm{~s}$ [4]. Although various factors are involved in the occurrence of these fractures, deterioration of bone strength due to aging and menopause is thought to be the most important factor $[1-3,5]$.

Bone strength is defined by structural and material properties. Structural properties include bone microstructure and geometry, and material properties include collagen, degrees of calcification, and microdamage [6]. Furthermore, bone microstructure is composed of trabecular and cortical bone microstructure, which is evaluated by various parameters, such as trabecular thickness, number, separation, connectivity density, cortical thickness, porosity, and so forth.

Bone microstructure has been analyzed by histological methods.
Today, microcomputed tomography (micro-CT) enables three-dimensional evaluation of microstructure non-destructively [7].

High-resolution peripheral quantitative computed tomography (HRpQCT) is a clinical imaging modality that can evaluate patients' bone microstructure in vivo. It also allows us to analyze the microstructure of bone samples ex vivo, similar to micro-CT [8]. While micro-CT is mainly used for small experimental animals, HR-pQCT can be used for large bone samples, such as the human cadaver spine [9-16]. It has been reported that HR-pQCT results in fewer errors in measurements of bone morphology compared to the histological method [17]. The latest model second-generation HR-pQCT scanner has a wide field of view (FOV) of $140 \mathrm{~mm}$ and a long scan length of $200 \mathrm{~mm}$ with one scan.

Finite element analysis (FEA) is a simulation method that enables us to predict bone strength under various conditions using image information without doing an actual mechanical breaking test. Previous studies have demonstrated that the predicted failure loads derived from FEA have a high correlation with the actual mechanical breaking test [11,12,19-21]. Micro-FEA using HR-pQCT or micro-CT images reflect

\footnotetext{
* Corresponding author at: Department of Orthopedic Surgery, Nagasaki University Graduate School of Biomedical Sciences, 1-7-1 Sakamoto, Nagasaki 852-8501, Japan.

E-mail address: kohchiba@estate.ocn.ne.jp (K. Chiba).
} 
trabecular and cortical bone structure and are able to simulate bone strength more precisely.

Bone microstructure is considered to be an important factor in defining vertebral strength. However, it is not yet fully understood how each bone microstructural parameter is related to the bone strength of vertebrae.

The purpose of this study was to investigate bone microstructural parameters that are most related to vertebral mechanical strength by analyzing bone microstructure and estimated bone strength using HRpQCT.

\section{Materials and methods}

\subsection{Specimens}

Three formalin-fixed cadavers of Japanese women with a mean age of 80.3 years (Sample 1: 76, Sample 2: 81, and Sample 3: 84 years) were selected. Cadavers with a history of bone metabolic disorders, vertebral fracture, spinal metastasis of a malignant tumor, and spinal implant insertion were excluded. The spine was separated from the skull and fifth lumbar vertebra, and the ribs were excised. Soft tissues were removed.

The study protocol was approved by the Institutional Review Board of Nagasaki University Graduate School of Biomedical Sciences (registration number: 15082033) and complied with the Declaration of Helsinki of 1975, revised in 2000.

\subsection{HR-pQCT scan}

The spine was scanned by a second-generation HR-pQCT (XtremeCT II, Scanco Medical, Brüttisellen, Switzerland).

Whole spines were sealed in a plastic bag and fixed to an acrylic plate, then scanned in four regions: cervical, upper thoracic, lower thoracic, and lumbar spine (Fig. 1a).

Scan settings were as follows: voltage $68 \mathrm{kVp}$, current $1470 \mu \mathrm{A}$, integration time $150 \mathrm{~ms}$, FOV $140 \mathrm{~mm}$, matrix $2304 \times 2304$, voxel size $60.7 \mu \mathrm{m}$, scan length $194 \mathrm{~mm}$, total number of slices 3192, and scan time $95 \mathrm{~min}$

Cervical vertebrae were excluded from the analysis because osteoporotic vertebral fractures are unlikely to occur at these levels. Sixteen vertebrae (T1-12 and L1-4) were analyzed in each sample, for a total of 48 vertebrae.

Image data of each vertebra were extracted from the full scan data, and analysis models were created. In this study, the superior and inferior endplates and posterior elements were removed following the procedures described by Pahr et al. [11] and Dall'Ara et al. [16] (Fig. 1b-d).

\subsection{Measurements of bone microstructure}

Each vertebra was separated into trabecular and cortical regions, and then the bone mineral density and microstructure were analyzed using bone microstructure analysis software (TRI/3D-BON, Ratoc System Engineering Co., Ltd., Tokyo, Japan) (Fig. 1e-g).

The process of segmentation is shown in Fig. 2. First, the image was binarized with a threshold value of $320 \mathrm{mg} / \mathrm{cm}^{3}$ (a). Then, the whole bone region was obtained by closing the bone marrow space (b). Next, only the bone marrow space was extracted with the same threshold value (c). The trabecular region is obtained by filling all trabecular bones in the bone marrow space (d). Trabecular bone microstructure was analyzed with the binarized images in the region (e). Finally, by subtracting the trabecular region from the whole bone region, the cortical region was obtained (f).

Measured trabecular bone parameters were: trabecular volumetric bone mineral density (Tb.vBMD), bone volume fraction (BV/TV), trabecular number (Tb.N), trabecular thickness (Tb.Th), trabecular separation (Tb.Sp), structure model index (SMI), connectivity density (Conn.D), and degree of anisotropy (DA). SMI is a parameter that evaluates trabecular bone morphology; when it is close to 0 , it appears plate-like in structure, whereas when it is close to 3 , it appears rod-like $[22,23]$. Conn.D provides data about the connectivity of the trabecular bone network by calculating the number of closed loops in a trabecular network [24,25]. DA is determined by the ratio between the primary and tertiary axes of the mean intercept length (MIL) ellipsoid (DA $=\mathrm{a}$ / c); a DA of 1.0 indicates that there is no anisotropy, and anisotropy increases as the value increases [10].

Cortical bone parameters were: cortical volumetric bone mineral density (Ct.vBMD), cortical thickness (Ct.Th), and cortical porosity (Ct.Po).

BMD $\left(\mathrm{mg} / \mathrm{cm}^{3}\right)$ was calculated from the X-ray attenuation value and a calibration curve obtained from a BMD phantom. Bone microstructural parameters were measured with an image binarized by $320 \mathrm{mg} / \mathrm{cm}^{3}$, and Tb.Th, Tb.Sp, and Ct.Th were analyzed by the direct measurement method.

The reproducibility of the microstructural parameters was analyzed by measuring three samples three times. The intraclass coefficient (ICC)
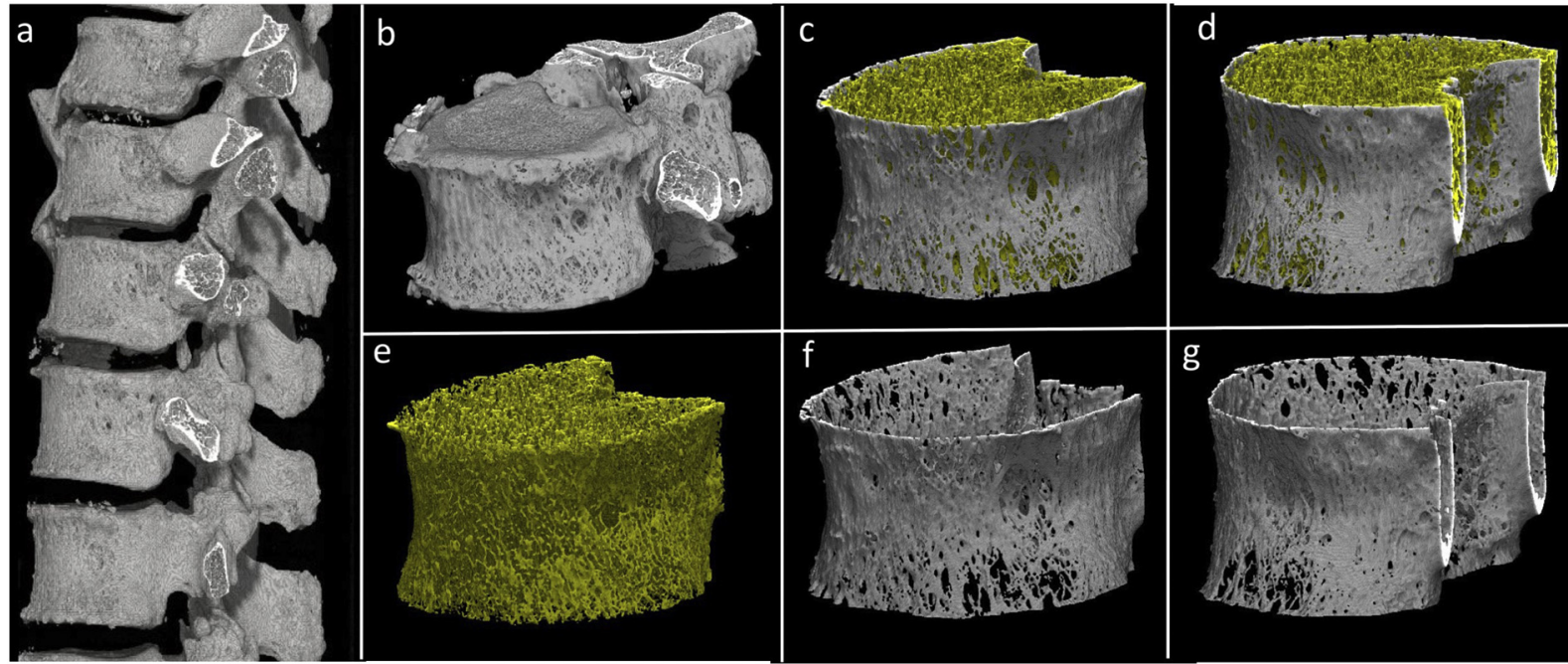

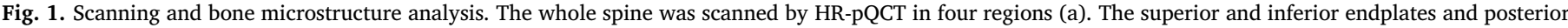
elements are removed from the vertebra (b, c, d). The bone microstructure is analyzed in trabecular and cortical regions (e, $\mathrm{f}$, $\mathrm{g}$ ). 

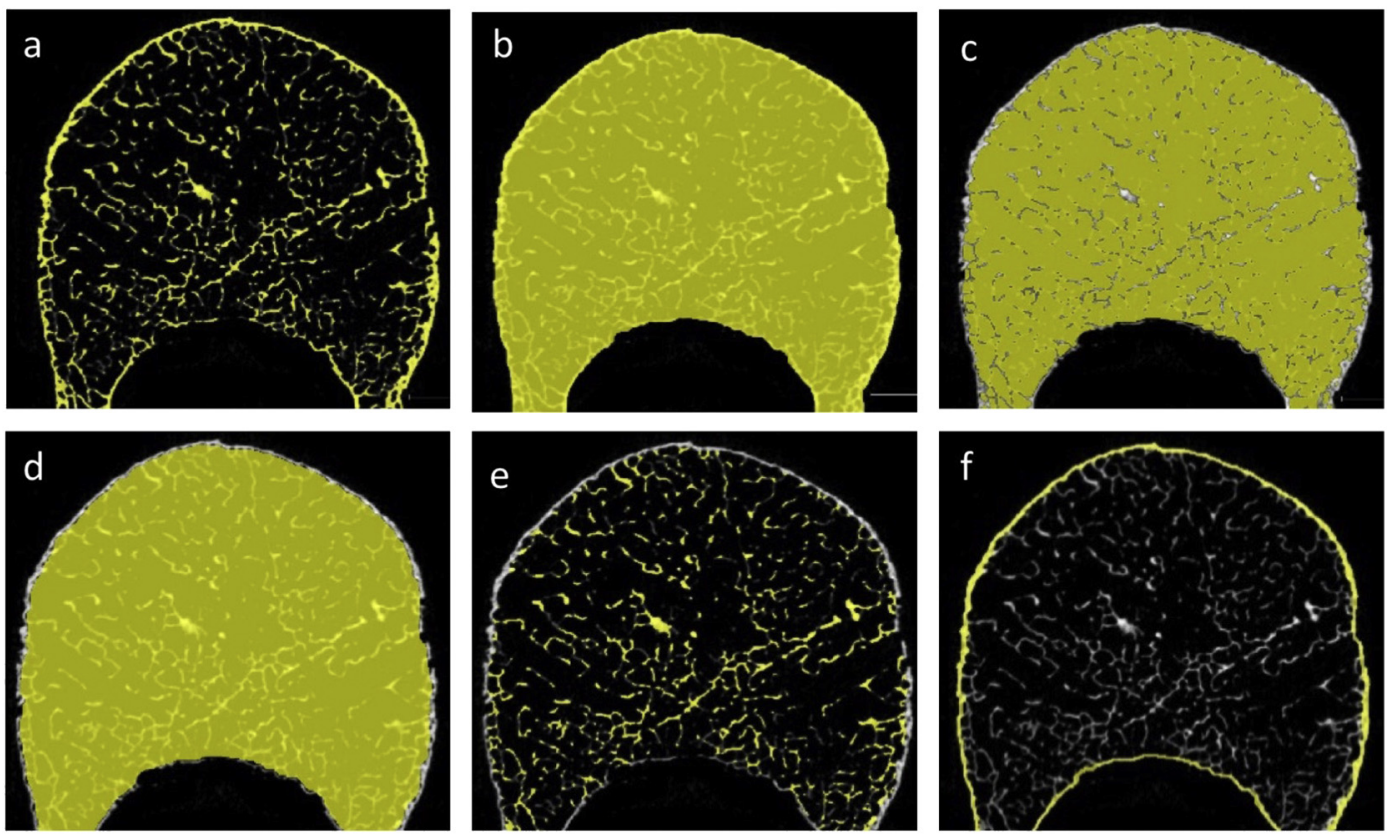

Fig. 2. Segmentation of trabecular and cortical regions. The image is binarized with a threshold value of $320 \mathrm{mg} / \mathrm{cm}^{3}$ (a). The whole bone region is obtained by closing the bone marrow space (b). The bone marrow space is extracted with the same threshold value (c). The trabecular region is obtained by filling all trabecular bones in the bone marrow space (d). Trabecular bone microstructure is analyzed with the binarized images in the area (e). By subtracting the trabecular region from the whole bone region, the cortical region is obtained (f).

of Tb.vBMD was 1.00, BV/TV 0.99, Tb.N 0.99, Tb.Th 1.00, Tb.Sp 0.99, SMI 0.89, Conn.D 1.00, DA 1.00, Ct.vBMD 0.99, Ct.Th 1.00, and Ct.Po 1.00 .

\subsection{Micro-FEA}

Micro-FEA was performed on all 48 vertebrae using FEA software (TRI/3D-FEM, Ratoc System Engineering Co. Ltd.). The voxel size of the images was converted to $120 \mu \mathrm{m}$, and unconnected trabeculae were deleted for voxel-based FEA. Young's modulus was applied to each voxel based on the BMD value [26], and Poisson's ratio was 0.3.

A linear FEA model was used for each vertebra with the following boundary and load settings: the inferior plane of a vertebra was fixed in all directions, and a uniaxial compression force corresponding to strain for $10 \%$ of vertebral height was applied to the superior plane (Fig. 3). Then, failure load and stiffness were calculated. A fracture was defined as occurring when $2 \%$ of the bone tissue was strained beyond a critical limit of $7.5 \%$ strain $[27,28]$.

Additionally, this FEA was performed in the model without trabecular bone in order to calculate failure load and stiffness of the cortical bone alone.

\subsection{Statistical analysis}

Univariate analysis was performed to analyze the correlations between all bone microstructural parameters and estimated vertebral strength using Pearson's correlation coefficients. Furthermore, multivariate regression analysis was performed to elucidate the bone

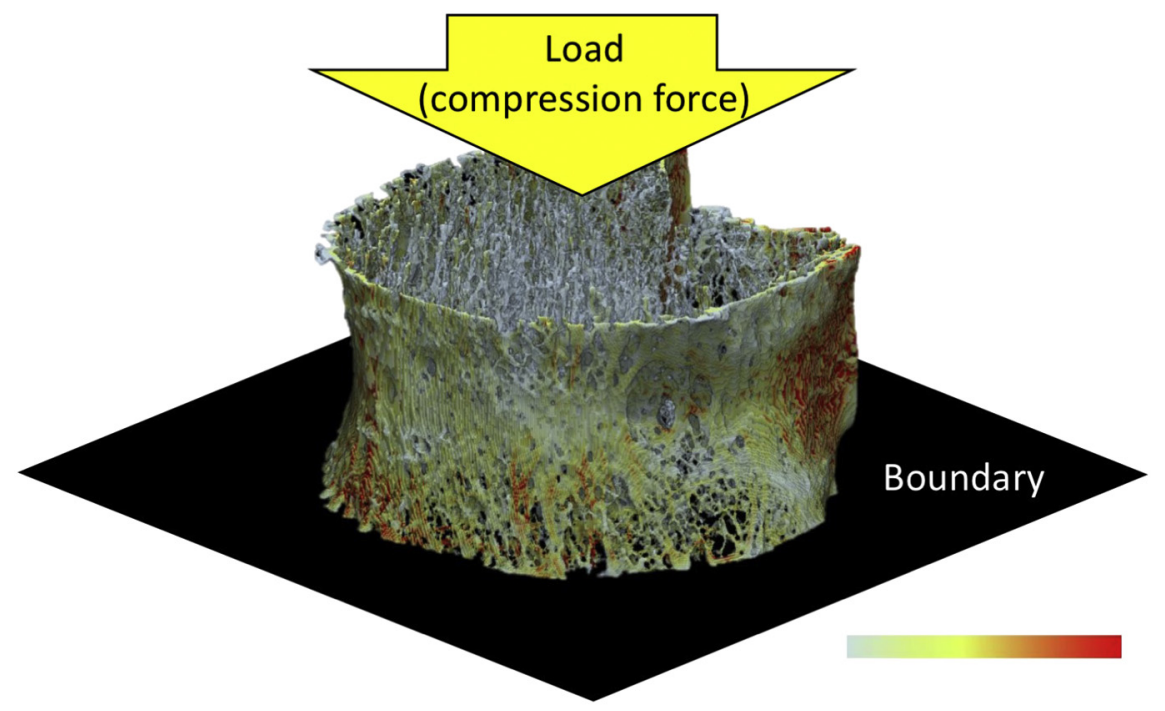

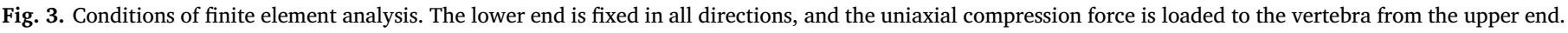
A color bar means the distribution of von Mises equivalent stress. 
Table 1

Summary of trabecular and cortical bone microstructure and estimated bone strength $(\mathrm{N}=48)$.

\begin{tabular}{lccccc}
\hline & & Mean & \multicolumn{2}{l}{ SD } & \multicolumn{2}{l}{ Min } & Max \\
\hline Tb.vBMD & $\left(\mathrm{mgHA} / \mathrm{cm}^{3}\right)$ & 63.7 & 31.3 & 31.9 & 158.1 \\
BV/TV & $(\%)$ & 12.6 & 5.9 & 6.7 & 30.7 \\
Tb.N & $(1 / \mathrm{mm})$ & 0.40 & 0.12 & 0.27 & 0.68 \\
Tb.Th & $(\mu \mathrm{m})$ & 232.1 & 31.1 & 178 & 328.1 \\
Tb.Sp & $(\mu \mathrm{m})$ & 836.8 & 195.9 & 461.5 & 1197.5 \\
SMI & & 2.3 & 0.3 & 1.3 & 2.8 \\
Conn.D & $\left(1 / \mathrm{mm}^{3}\right)$ & 1.3 & 0.8 & 0.5 & 3.5 \\
DA & & 1.5 & 0.2 & 1.1 & 1.9 \\
Ct.vBMD & $\left(\mathrm{mgHA} / \mathrm{cm}^{3}\right)$ & 499.1 & 75.8 & 344.2 & 631.1 \\
Ct.Th & $(\mu \mathrm{m})$ & 512.7 & 130 & 327.7 & 913.6 \\
Ct.Po & $(\%)$ & 16.3 & 3.2 & 9.1 & 24.7 \\
Failure Load $(\mathrm{Ct}$. & $(\mathrm{N})$ & 2774 & 1927 & 471 & 9833 \\
$\quad$ alone) & & $(1114)$ & $(1017)$ & $(121)$ & $(4249)$ \\
Stiffness (Ct. & $(\mathrm{N} / \mathrm{mm})$ & 8617 & 6261 & 1133 & 21610 \\
$\quad$ alone) & & $(3220)$ & $(3396)$ & $(251)$ & $(12255)$ \\
\hline
\end{tabular}

Tb.vBMD: trabecular volumetric BMD, BV/TV: bone volume fraction, Tb.N: trabecular number, Tb.Th: trabecular thickness.

Tb.Sp: trabecular separation, SMI: structure model index, Conn.D: connectivity density, DA: degree of anisotropy.

Ct.vBMD: cortical volumetric BMD, Ct.Th: cortical thickness, Ct.Po: cortical porosity.

microstructural parameters most strongly related to estimated bone strength by Ridge regression analysis (JMP ${ }^{\circledR} 13$, SAS Institute Inc., Cary, NC, USA).

\section{Results}

\subsection{Bone microstructure and estimated bone strength}

The results of trabecular and cortical bone microstructure and estimated bone strength are shown in Table 1.

Regarding trabecular parameters, Tb.N was low at $0.4 / \mathrm{mm}$, and Tb.Sp was wide at $837 \mu \mathrm{m}$, showing disappearance of trabeculae. As a result, Tb.vBMD and BV/TV were remarkably low. SMI (0 to 3) was 2.3, meaning a rod-like structure. As to cortical parameters, Ct.Th was extremely thin at $512.7 \mu \mathrm{m}$, and Ct.Po was high at $16.3 \%$.

The results of the uniaxial compression simulation showed a mean failure load of $2774 \mathrm{~N}$ and estimated stiffness of $8617 \mathrm{~N} / \mathrm{mm}$. As to the cortical FEA model, the mean failure load was $1114 \mathrm{~N}$, and estimated stiffness was $3220 \mathrm{~N} / \mathrm{mm}$.

\subsection{Correlation analysis}

Table 2 shows the Pearson's correlation coefficients between trabecular and cortical bone microstructural parameters and estimated vertebral strength. The parameters that were strongly correlated with estimated failure load were Tb.Th, Ct.Th, and Ct.vBMD ( $r=0.82,0.89$, 0.67). The parameters that were strongly correlated with estimated stiffness were also Tb.Th, Ct.Th, and Ct.vBMD ( $r=0.81,0.78,0.79$ ).

Table 3 shows the results of multivariate regression analysis by the Ridge regression model. The bone microstructural parameters that correlated with estimated failure load were Tb.vBMD, BV/TV, Tb.N, and Ct.Th. The parameters that were correlated with estimated stiffness were Tb.vBMD and BV/TV. Linear regression models of these parameters and failure load are shown in Fig. 4.

\section{Discussion}

The purpose of this study was to investigate bone microstructural parameters that define the bone strength of the vertebra. It was found that both trabecular and cortical factors were significantly correlated with the estimated failure load in elderly women.

As shown in Table 1, bone samples that were analyzed in this study had deteriorated bone microstructure, with an average Tb.vBMD of $63.7 \mathrm{mgHA} / \mathrm{cm}^{3}$ and Ct.vBMD of $499 \mathrm{mgHA} / \mathrm{cm}^{3}$. It has been reported that the average Tb.vBMD and Ct.vBMD at the distal radius of typical 80 -year-old women are approximately $100 \mathrm{mgHA} / \mathrm{cm}^{3}$ and $760-850 \mathrm{mgHA} / \mathrm{cm}$, respectively $[3,29,30]$. Considering that the distal radius is not a weight-bearing bone, it can be said that both trabecular and cortical bone in this series had remarkably low vBMD.

As shown in Table 2, on univariate analysis, bone microstructural parameters that had particularly strong correlations with estimated failure load were Tb.Th and Ct.Th ( $r=0.82$ and 0.89 , respectively). On the other hand, when looking at the correlations between trabecular and cortical bone parameters, some of them had strong correlations with each other, and $\mathrm{Tb}$.Th was strongly correlated with Ct.Th $(r=0.86)$. To identify the bone microstructural parameters that were authentically correlated with vertebral strength, multivariate regression analysis was necessary, and Ridge regression analysis was performed to eliminate multicollinearity.

As shown in Table 3, on multivariate regression analysis, Tb.vBMD, BV/TV, Tb.N, and Ct.Th were significantly correlated with estimated failure load.

First, these results mean that the trabecular component is definitely the primary factor to explain vertebral strength. Previous studies have proven that vertebral strength is mainly dependent on trabecular bone [14,31-33]. Lu et al. conducted mechanical compression tests of T12 vertebrae in elderly women ( $\mathrm{N}=20$, average age: 80 years), and they reported that the factor that was most correlated with failure load was Tb.vBMD (Pearson's correlation coefficient, $r=0.66$ ), whereas the correlation with Ct.Th was low $(r=0.35)$ [14]. Similarly, Wegrzyn

Table 2

Correlation coefficients between trabecular and cortical bone microstructure and estimated bone strength.

\begin{tabular}{|c|c|c|c|c|c|c|c|c|c|c|c|c|c|}
\hline & Tb.vBMD & $\mathrm{BV} / \mathrm{TV}$ & Tb.N & Tb.Th & Tb.Sp & SMI & Conn.D & DA & Ct.vBMD & Ct.Th & Ct.Po & Failure Load & Stiffness \\
\hline Tb.vBMD & 1 & & & & & & & & & & & & \\
\hline $\mathrm{BV} / \mathrm{TV}$ & $0.99^{* *}$ & 1 & & & & & & & & & & & \\
\hline Tb.N & $0.90^{* *}$ & $0.93^{* * *}$ & 1 & & & & & & & & & & \\
\hline Tb.Th & $0.68^{* *}$ & $0.63^{\text {** }}$ & $0.33^{*}$ & 1 & & & & & & & & & \\
\hline Tb.Sp & $-0.92^{* *}$ & $-0.93^{* *}$ & $-0.90^{* *}$ & $-0.57^{* * *}$ & 1 & & & & & & & & \\
\hline SMI & $-0.85^{* *}$ & $-0.88^{* *}$ & $-0.92^{* * *}$ & -0.25 & $0.76^{* *}$ & 1 & & & & & & & \\
\hline Conn.D & $0.95^{* *}$ & $0.97^{* *}$ & $0.98^{* *}$ & $0.44^{* *}$ & $-0.93^{* *}$ & $-0.90^{* *}$ & 1 & & & & & & \\
\hline DA & $-0.41^{* *}$ & $-0.39^{* *}$ & -0.23 & $-0.59^{* *}$ & $0.47^{* * *}$ & 0.15 & $-0.30^{*}$ & 1 & & & & & \\
\hline Ct.vBMD & $0.37^{*}$ & $0.32^{*}$ & 0.08 & $0.76^{* *}$ & $-0.39^{* * *}$ & 0.12 & 0.20 & $-0.54^{* *}$ & 1 & & & & \\
\hline Ct.Th & $0.36^{*}$ & $0.31^{*}$ & 0.05 & $0.86^{* *}$ & -0.32 & 0.09 & 0.13 & $-0.52^{* *}$ & $0.85^{* *}$ & 1 & & & \\
\hline Ct.Po & -0.25 & -0.24 & -0.11 & $-0.34 *$ & 0.26 & 0.07 & -0.18 & $0.37^{* *}$ & $-0.67^{* *}$ & $-0.39^{* * *}$ & 1 & & \\
\hline Failure Load & $0.45^{* *}$ & $0.40^{* *}$ & 0.17 & $0.82^{* *}$ & $-0.40^{* *}$ & -0.07 & 0.23 & $-0.49^{* *}$ & $0.67^{* *}$ & $0.89^{* *}$ & -0.17 & 1 & \\
\hline Stiffness & $0.48^{* *}$ & $0.43^{* *}$ & 0.19 & $0.81^{* *}$ & $-0.46^{* * *}$ & -0.07 & $0.30^{*}$ & $-0.58^{* *}$ & $0.79^{* *}$ & $0.78^{* * *}$ & -0.34 & $0.86^{* *}$ & 1 \\
\hline
\end{tabular}

\footnotetext{
* Pearson's correlation coefficient: $P<0.05$.
}

** Pearson's correlation coefficient : $P<0.01$. 
Table 3

Multivariate analysis of trabecular and cortical bone microstructure with estimated bone strength.

\begin{tabular}{|c|c|c|c|c|c|c|}
\hline & \multicolumn{3}{|l|}{ Failure Load } & \multicolumn{3}{|l|}{ Stiffness } \\
\hline & Coefficient & $95 \% \mathrm{CI}$ & $P$ value & Coefficient & $95 \% \mathrm{CI}$ & $P$ value \\
\hline Tb.vBMD & 404.3 & $184.4,624.2$ & $0.0003^{* *}$ & 1036.5 & $253.8,1819.2$ & $0.009^{* *}$ \\
\hline $\mathrm{BV} / \mathrm{TV}$ & -2008.3 & $-3235.2,-781.5$ & $0.0013^{* * *}$ & -5406.8 & $-9991.6,-822$ & $0.02 *$ \\
\hline Tb.N & $14,697.5$ & 2215.5, 27,179 & $0.0210^{*}$ & $29,329.1$ & $-19,427,78,085.2$ & 0.23 \\
\hline Tb.Th & -17.3 & $-58.7,24$ & 0.41 & 1.8 & $-187.9,191.6$ & 0.98 \\
\hline Tb.Sp & -1.1 & $-6.1,3.9$ & 0.67 & -1.9 & $-20,16.2$ & 0.83 \\
\hline SMI & 54.6 & $-5520.1,5629.2$ & 0.98 & -9300.5 & $-30,333.2,11,732$ & 0.38 \\
\hline Conn.D & -2211.5 & $-5713.3,1290.1$ & 0.21 & -6304.9 & $-17,736,5126$ & 0.27 \\
\hline DA & -729.5 & $-1911.4,452.4$ & 0.22 & -5243.9 & $-11,721.31233 .3$ & 0.11 \\
\hline Ct.vBMD & -4.66 & $-25.7,16.4$ & 0.66 & 78.8 & $-12.5,170.3$ & 0.09 \\
\hline CtTh & 12.1 & $3.4,20.8$ & $0.0063^{* *}$ & -11.1 & -44.722 .4 & 0.51 \\
\hline Ct.Po & 49.4 & $-149.2,248.1$ & 0.62 & 624.4 & $-252.8,1501.8$ & 0.16 \\
\hline
\end{tabular}

* Ridge regression analysis: $P<0.05$.

** Ridge regression analysis: $P<0.01$.

(a) Failure Load (N)

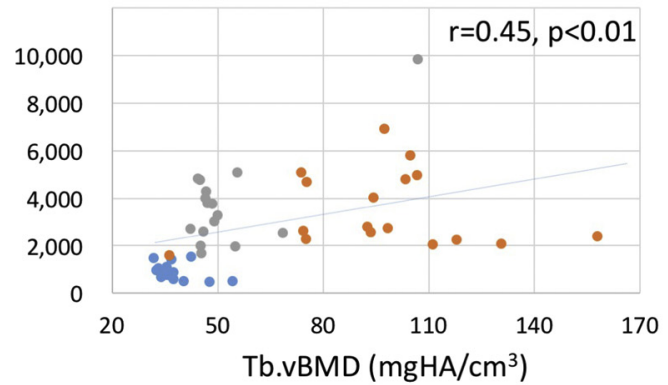

(c) Failure Load (N)

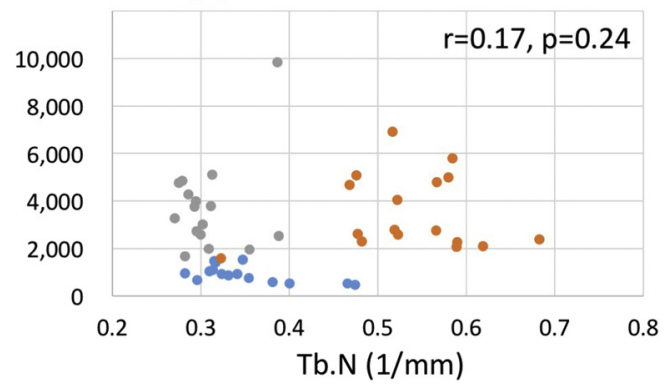

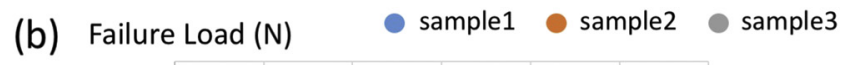

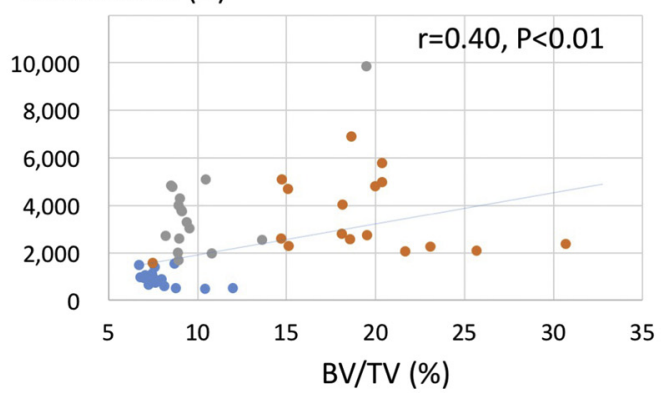

(d) Failure Load (N)

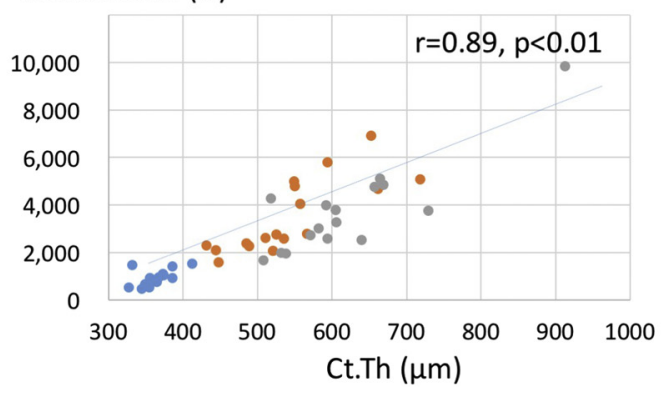

Fig. 4. Linear regression models between bone microstructural parameters and estimated failure load (a: Tb.vBMD, b: BV/TV, c: Tb.N, d: Ct.Th).

et al. performed a compression breaking test of the lumbar spine $(\mathrm{N}=17$, average age: 76 years) and reported that BV/TV was strongly correlated with vertebral strength (Pearson's correlation coefficient, $r=0.69)$, but the correlation with Ct.Th was low $(r=0.24)$ [34]. Fields et al. carried out micro-CT-based FEA of T9 vertebrae in elderly people ( $\mathrm{N}=22$, average age: 81 years) and reported that vertebral strength was strongly correlated with SMI of trabecular bone (Pearson's correlation coefficient, $r=-0.76$ ), and Ct.Th was also correlated $(r=0.50)$ [33].

Second, the results of the present study mean that the cortical component is also an important factor contributing to vertebral strength. Some previous studies reported that the contributions of trabecular and cortical bone to vertebral strength differed depending on the age of the subjects. Rockoff et al. performed compression tests on the lumbar vertebrae ( $\mathrm{N}=50$, age: 10 s to $80 \mathrm{~s})$, compared with a cortical bone-removed model and a trabecular bone-removed model, and they reported that cortical bone contributed approximately $65 \%$ of vertebral body strength in persons $\geq 40$ years old [35]. Blaine et al. performed QCT-based FEA of vertebrae (T10 and L3), compared between younger people ( $\mathrm{N}=30$, average age: 38 years) and elderly people ( $N=30$, average age: 78 years), and they reported that the percentage of contribution of the cortical bone to vertebral strength was approximately $40 \%$ for younger people, but over $50 \%$ for elderly people [36]. In this study, the estimated failure loads of the total and cortical bone were $2774 \mathrm{~N}$ and $1114 \mathrm{~N}$, respectively. It was considered that cortical bone contributed to almost $40 \%$ of the bone strength of the vertebral body.

It is obvious that the age-related changes of bone microstructure are different between trabecular and cortical bones [29,30,37]. Blaine et al. compared BMD of the vertebral bone in younger people $(\mathrm{N}=30$, average age: 38 years) and elderly people $(\mathrm{N}=30$, average age: 78 years) and reported that the decrease of cortical bone was approximately $30 \%$, while that of trabecular bone was approximately $40 \%$ [36]. The deterioration of the trabecular bone with age would cause a relatively increased contribution of the cortical bone to vertebral strength. This might be one of the reasons why a cortical factor (Ct.Th) correlated with estimated failure load, as did trabecular factors (Tb.vBMD, BV/TV, Tb.N).

\subsection{Limitations}

This study has several limitations. A total of 48 vertebrae were 
obtained from only three cadavers. T1-12 and L1-4 vertebrae were analyzed in each cadaver because osteoporotic fractures occur at various levels. However, different cross-sectional areas and heights of vertebrae would affect estimated bone strength.

Samples were fixed in formalin. Lochmüller et al. [38] reported that the effect of formalin fixation on BMD was $<9 \%$. Baum et al. [39] performed QCT-based FEA using human frozen specimens and formalin-fixed specimens and reported that there was no effect of formalin fixation on trabecular microstructure and vertebral strength.

Since the voxel size of the CT images was $60 \mu \mathrm{m}$, trabecular bones $<60 \mu \mathrm{m}$ were ignored in this study.

Endplates, posterior elements, intervertebral discs, ligaments, and muscles were excluded in the FEA model. Although the quality of collagen is also an important factor, it cannot be evaluated by CT. Due to the limitations of the software, FEA was performed by a linear model with voxel size of $120 \mu \mathrm{m}$ in the present study. Also, because of the ethical limitations of our facility, mechanical tests were not performed to verify the FEA model.

It is necessary to apply bending forces in addition to compression force under various conditions, because the global balance of the whole spine changes due to its degeneration, which affects load vectors to the vertebrae [40].

\section{Conclusion}

The relationship between vertebral bone microstructure and estimated bone strength was analyzed in cadaveric spines using secondgeneration HR-pQCT. Bone microstructural parameters that were correlated with the failure load were Tb.vBMD, BV/TV, Tb.N, and Ct.Th. In addition to trabecular bone, cortical thickness also contributes significantly to vertebral strength in elderly women with highly deteriorated bone microstructure.

\section{Acknowledgment}

The authors would like to thank Shuntaro Sato for advising on the statistical analysis.

\section{References}

[1] S.R. Cummings, L.J. Melton, Osteoporosis I: epidemiology and outcomes of osteoporotic fractures, Lancet 359 (2002) 1761-1767.

[2] Cooper Cyrus, J. Atkinson Elizabeth, J. Jacobsen Steven, et al., Population-based study of survival after osteoporotic fractures, Am. J. Epidemiol. 137 (1993) 1001-1005.

[3] A.A. Edidin, K.L. Ong, E. Lau, et al., Morbidity and mortality after vertebral fractures, Spine (Phila Pa 1976) 40 (2015) (Gerling).

[4] S. Fujiwara, F. Kasagi, N. Masunari, et al., Fracture prediction from bone mineral density in Japanese men and women, J. Bone Miner. Res. 18 (8) (2003 Aug) $1547-1553$.

[5] J.R. Tucci, R.P. Tonino, R.D. Emkey, et al., Effect of three years of oral alendronate treatment in postmenopausal women with osteoporosis, Am. J. Med. 101 (1996) 488-501.

[6] E. Donnelly, Methods for assessing bone quality: a review, Clin. Orthop. Relat. Res. 469 (8) (2011) 2128-2138.

[7] P. Ruegsegger, B. Koller, R. Muller, A microtomographic system for the nondestructive evaluation of bone architecture, Calcif. Tissue Int. 58 (1996) 24-29.

[8] J.A. MacNeil, S.K. Boyd, Accuracy of high-resolution peripheral quantitative computed tomography for measurement of bone quality, Med. Eng. Phys. 29 (2007) 1096-1105.

[9] K. Chiba, A.J. Burghardt, M. Osaki, et al., Three-dimensional analysis of subchondral cysts in hip osteoarthritis: an ex vivo HR-pQCT study, Bone 66 (2014) 140-145.

[10] K. Chiba, A.J. Burghardt, M. Osaki, et al., Heterogeneity of bone microstructure in the femoral head in patients with osteoporosis: an ex vivo HR-pQCT study, Bone 56 (2013) 139-146.

[11] D.H. Pahr, J. Schwiedrzik, E. Dall'Ara, et al., Clinical versus pre-clinical FE models for vertebral body strength predictions, J. Mech. Behav. Biomed. Mater. 33 (2014) 76-83.

[12] Y. Lu, G. Maquer, O. Museyko, et al., Finite element analyses of human vertebral bodies embedded in polymethylmethalcrylate or loaded via the hyperelastic intervertebral disc models provide equivalent predictions of experimental strength, J. Biomech. 47 (2014) 2512-2516.

[13] T. Baum, M. Gräbeldinger, C. Räth, et al., Trabecular bone structure analysis of the spine using clinical MDCT: can it predict vertebral bone strength? J. Bone Miner. Metab. 32 (2014) 56-64.

[14] Y. Lu, M. Krause, N. Bishop, et al., The role of patient-mode high-resolution peripheral quantitative computed tomography indices in the prediction of failure strength of the elderly women's thoracic vertebral body, Osteoporos. Int. 26 (2014) 237-244.

[15] J. Wegrzyn, J.P. Roux, M.E. Arlot, et al., Role of trabecular microarchitecture and its heterogeneity parameters in the mechanical behavior of ex vivo human L3 vertebrae, J. Bone Miner. Res. 25 (2010) 2324-2331.

[16] E. Dall'Ara, R. Schmidt, D. Pahr, et al., A nonlinear finite element model validation study based on a novel experimental technique for inducing anterior wedge-shape fractures in human vertebral bodies in vitro, J. Biomech. 43 (2010) 2374-2380.

[17] R. Müller, M. Hahn, M. Vogel, et al., Morphometric analysis of noninvasively assessed bone biopsies: comparison of high-resolution computed tomography and histologic sections, Bone 18 (1996) 215-220.

[19] J.S. Bauer, I. Sidorenko, D. Mueller, et al., Prediction of bone strength by $\mu c T$ and MDCT-based finite-element-models: how much spatial resolution is needed? Eur. J. Radiol. 83 (2014) e36-e42.

[20] J.M. Buckley, L. Cheng, K. Loo, et al., Quantitative computed tomography-based predictions of vertebral strength in anterior bending, Spine (Phila Pa 1976) (32) (2007) 1019-1027.

[21] E. Dall'Ara, D. Pahr, P. Varga, et al., QCT-based finite element models predict human vertebral strength in vitro significantly better than simulated DEXA, Osteoporos. Int. 23 (2012) 563-572.

[22] M. Sode, A.J. Burghardt, R.A. Nissenson, et al., Resolution dependence of the nonmetric trabecular structure indices, Bone 42 (2008) 728-736.

[23] T. Hildebrand, P. Rüegsegger, Quantification of bone microarchitecture with the structure model index, Comput. Methods Biomech. Biomed. Engin. 1 (1) (1997) 15-23.

[24] X.S. Liu, X.H. Zhang, K.K. Sekhon, et al., High-resolution peripheral quantitative computed tomography can assess microstructural and mechanical properties of human distal tibial bone, J. Bone Miner. Res. 25 (4) (2010) 746-756.

[25] A. Odgaard, H.J. Gundersen, Quantification of connectivity in cancellous bone, with special emphasis on 3-D reconstructions, Bone 14 (2) (1993) 173-182.

[26] D.R. Carter, W.C. Hayes, The compressive behavior of bone as a two phase porous structure, J. Bone Joint Surg. Am. 59 (7) (1977 Oct) 954-962.

[27] W. Pistoia, B. van Rietbergen, E.-M. Lochmüller, et al., Estimation of distal radius failure load with micro-finite element analysis models based on three-dimensional peripheral quantitative computed tomography images, Bone 30 (2002) 842-848.

[28] S. Boutroy, B. Van Rietbergen, E. Sornay-Rendu, et al., Finite element analysis based on in vivo HR-pQCT images of the distal radius is associated with wrist fracture in postmenopausal women, J. Bone Miner. Res. 23 (2007) 392-399.

[29] V.W.Y. Hung, T.Y. Zhu, W.H. Cheung, et al., Age-related differences in volumetric bone mineral density, microarchitecture, and bone strength of distal radius and tibia in Chinese women: a high-resolution pQCT reference database study, Osteoporos. Int. 26 (2015) 1691-1703.

[30] S. Hansen, V. Shanbhogue, L. Folkestad, et al., Bone microarchitecture and estimated strength in 499 adult Danish women and men: a cross-sectional, populationbased high-resolution peripheral quantitative computed tomographic study on peak bone structure, Calcif. Tissue Int. 94 (2014) 269-281.

[31] J. Hazrati Marangalou, F. Eckstein, V. Kuhn, et al., Locally measured microstructural parameters are better associated with vertebral strength than whole bone density, Osteoporos. Int. 25 (2014) 1285-1296.

[32] S.K. Eswaran, A. Gupta, M.F. Adams, T.M. Keaveny, Cortical and trabecular load sharing in the human vertebral body, J. Bone Miner. Res. 21 (2006) 307-314.

[33] A.J. Fields, S.K. Eswaran, M.G. Jekir, et al., Role of trabecular microarchitecture in whole-vertebral body biomechanical behavior, J. Bone Miner. Res. 24 (2009) 1523-1530.

[34] J. Wegrzyn, J.P. Roux, D. Farlay, et al., The role of bone intrinsic properties measured by infrared spectroscopy in whole lumbar vertebra mechanics: organic rather than inorganic bone matrix? Bone 56 (2013) 229-233.

[35] S.D. Rockoff, E. Sweet, J. Bleustein, The relative contribution of trabecular and cortical bone to the strength of human lumbar vertebrae, Calcif. Tissue Res. 3 (1969) 163-175.

[36] B.A. Christiansen, D.L. Kopperdahl, D.P. Kiel, et al., Mechanical contributions of the cortical and trabecular compartments contribute to differences in age-related changes in vertebral body strength in men and women assessed by QCT-based finite element analysis, J. Bone Miner. Res. 26 (2011) 974-983.

[37] J.S. Thomsen, E.N. Ebbesen, L. Mosekilde, Zone-dependent changes in human vertebral trabecular bone: clinical implications, Bone 30 (2002) 664-669.

[38] E.M. Lochmüller, N. Krefting, D. Bürklein, et al., Effect of fixation, soft-tissues, and scan projection on bone mineral measurements with dual energy, X-ray absorptiometry (DXA), Calcif. Tissue Int. 68 (2001) 140-145.

[39] T. Baum, E. Grande Garcia, R. Burgkart, et al., Osteoporosis imaging: effects of bone preservation on MDCT-based trabecular bone microstructure parameters and finite element models, BMC Med. Imaging 15 (2015) 22.

[40] A.G. Bruno, K. Burkhart, B. Allaire, et al., Spinal loading patterns from biomechanical modeling explain the high incidence of vertebral fractures in the thoracolumbar region, J. Bone Miner. Res. 32 (2017) 1282-1290. 